\title{
Graves' disease overlapping with chronic hepatitis $B$ and methimazole-induced liver injury and autoimmune hepatitis: a case report
}

\author{
Meifang Zheng ${ }^{1}$, Shiyuan Cui ${ }^{1}$, Wei Zhang ${ }^{1}$, David R. Brigstock ${ }^{2}$ and Runping Gao ${ }^{1 *}$ (C)
}

\begin{abstract}
Background: Liver injury related to Graves' Disease (GD) includes hepatotoxicity of thyroid hormone excess, druginduced liver injury, and changes resulting from concomitant liver disease. Methimazole (MMI) has been shown to induce several patterns of liver injury. However, the diagnosis and treatment of autoimmune hepatitis (AlH) overlapping with either GD or chronic hepatitis B are challenging.

Case presentation: A 35-year-old man from China presented with a two-year history of GD and a 10-day history of progressive jaundice. He had taken MMl for two months and discontinuing treatment due to liver toxicity 1 year ago and for another 6 days 20 days prior to hospitalization. The patient was diagnosed with GD overlapping with chronic hepatitis B and MMI-induced liver injury with early stage of acute-on-chronic liver failure on admission. However, the elevated aminotransferase and bilirubin levels could not be controlled after correction of liver failure and effective control of HBV replication and hyperthyroidism by daily oral entecavir and one-time oral administration of 131-iodine. The patient underwent liver biopsy on the 43rd day of hospitalization, showing HBsAg expression on the membrane of hepatocytes and typical histopathological characteristics of AlH. He was finally diagnosed with GD overlapping with chronic hepatitis B and MMI-induced liver injury and AlH. The elevated aminotransferase and bilirubin completely returned to normal by 3-month glucocorticoid therapy and continuous entecavir treatment and there was no recurrence during a 6-month follow-up, suggesting that AlH in this patient is different from classical AlH or GD-associated $\mathrm{AlH}$.
\end{abstract}

Conclusions: GD together with AlH is a complex and difficult subject. It needs to be clarified whether MMI or HBV can act as a trigger for AlH in this patient.

Keywords: Graves' disease, Hepatitis B virus, Glucocorticoids, Methimazole, Case report

\section{Background}

Liver dysfunction related to hyperthyroidism includes abnormalities associated with the effects of thyroid hormone excess, drug-induced liver injury, and changes resulting from concomitant liver disease [1, 2]. Methimazole (MMI) is the first-line anti-thyroid agent used in

\footnotetext{
*Correspondence: gao runping@126.com

${ }^{1}$ Department of Hepatic Biliary Pancreatic Medicine, First Hospital of Jilin

University, 71 Xinmin Avenue, Changchun 130021, Jilin, China

Full list of author information is available at the end of the article
}

clinical practice for management of Graves' disease (GD) [2] which is the most common cause of hyperthyroidism [3]. MMI is also associated with a certain degree of hepatic injury, although its adverse effects are less severe than those of propylthiouracil (PTU) [1]. Clinically, the characteristics of MMI-induced hepatotoxicity are variable and include necro-inflammation, granulomas, and/ or steatosis, with a dominant pattern of cholestasis [3-5].

MMI has been demonstrated to decrease adenosine triphosphate (ATP) levels, to increase reactive oxygen species (ROS) content, and to damage mitochondrial 
membranes in the liver of mouse models [6]. However, MMI seems to be a novel mitochondrial protecting agent in vitro, suggesting that MMI bioactivation and reactive metabolite formation potentially contribute to liver injury [6]. Recently a metabolomic study in patients with GD revealed that monoamine oxidase inhibition, ROS production, mitochondrial dysfunction, and DNA disruption might contribute to MMI-induced hepatotoxicity [7]. It has also been reported that MMI induces autoimmune diseases including antineutrophil cytoplasmic antibody (ANCA)-positive microscopic polyangiitis and insulin autoimmune syndrome (IAS) $[8,9]$. Allergic reactions are considered to contribute to MMI-induced liver injury and cholestatic jaundice, and some of these cases have been successfully controlled by glucocorticoids $[4$, 10]. A case of GD complicated with MMI-induced liver injury with AIH histological features has been described, implying MMI may act as a trigger for AIH [11]. GD overlapping with chronic hepatitis $\mathrm{B}(\mathrm{CHB})$ is not uncommon in China. AIH superimposed on $\mathrm{CHB}$ has also been reported. However, there are different opinions as to whether hepatitis B virus (HBV) functions as a trigger for AIH $[12,13]$.

In this report, a case from Northeast China of GD overlapping with $\mathrm{CHB}$ and MMI-induced liver injury and $\mathrm{AIH}$ is described. This rare clinical case has never been reported in literature.

\section{Case presentation}

A 35-year-old man was admitted to hospital due to progressive jaundice for 10 days. He had a two-year history of GD. MMI $30 \mathrm{mg} /$ day was initiated one year ago, but was discontinued after two months due to symptoms of fatigue, palpitations and diarrhea and abnormal liver function tests (alanine aminotransferase, ALT $268 \mathrm{U} / \mathrm{L}$, total bilirubin, TBil $54.7 \mu \mathrm{mol} / \mathrm{L}$ ), and his liver function was normal one month later. MMI $30 \mathrm{mg} /$ day was taken again for 6 days 20 days prior to hospitalization but discontinued because of same symptoms. Serum HBsAg was positive and the liver function test revealed increased levels of aspartate aminotransferase (AST, 722.3 U/L), alanine aminotransferase (ALT, 818.4 U/L), alkaline phosphatase (ALP, $192 \mathrm{U} / \mathrm{L}$ ), total bilirubin (TBil, $447.7 \mu \mathrm{mol} / \mathrm{L}$ ) in the local hospital one week before. The patient had no histories of alcohol, tobacco, hypertension and heart disease and his serum HBsAg had not been detected until one week prior to hospitalization. Physical examination on admission to this hospital showed a blood pressure of $135 / 78 \mathrm{mmHg}$, a heart rate of $100 \mathrm{bpm}$, intense jaundice, mild diffuse thyromegaly. The liver and spleen were not palpable. Liver function test were as follows: AST 133.9 U/L, ALT 137.7 U/L, ALP 149.3 U/L, cholinesterase (CHE) $2676 \mathrm{U} / \mathrm{L}$, albumin (Alb) $30.4 \mathrm{~g} / \mathrm{L}$,
TBil $831.9 \mu \mathrm{mol} / \mathrm{L}$, direct bilirubin (DBil) $554.6 \mu \mathrm{mol} / \mathrm{L}$, total bile acid (TBA) $493.5 \mu \mathrm{mol} / \mathrm{L}$. Fasting blood glucose was $2.8 \mathrm{mmol} / \mathrm{L}$. Prothrombin activity (PTA) was $38 \%$ and international normalized ratio (INR) was 1.51 . Thyroid stimulating hormone (TSH) was $0.0025 \mu \mathrm{IU} / \mathrm{mL}$, free triiodothyronine (FT3) was more than $30.7 \mathrm{pmol} / \mathrm{L}$ (upper limit of normal (ULN), $6.01 \mathrm{pmol} / \mathrm{L}$ ), anti-thyroglobulin antibody (TgAb) was positive. Tests for anti$\mathrm{HAV}$, anti-HEV, anti-EBV, anti-CMV IgMs and anti-HCV IgG were negative. Tests for $\mathrm{HBsAg}, \mathrm{HBeAg}$ and anti$\mathrm{HBc}$ were positive. HBV DNA was $3.28 \times 10^{4} \mathrm{IU} / \mathrm{mL}$. The titer of the antinuclear antibody (ANA) was 1: 320 . IgG was $22.17 \mathrm{~g} / \mathrm{L}$ (ULN, 17.4 g/L) and IgA and IgM were within normal limits. $2 \mathrm{~h}$ and $24 \mathrm{~h}$ radioiodine uptakes were $37.9 \%$ and $41.3 \%$ respectively. CT showed normal liver morphology and mild splenomegaly. Ultrasound showed a diffusely enlarged thyroid. The echocardiogram was normal.

The patient was preliminarily diagnosed with GD complicated with $\mathrm{CHB}, \mathrm{MMI}$-induced liver injury and earlystage acute-on-chronic liver failure (ACLF). Entecavir (ETV) $(0.5 \mathrm{mg} / \mathrm{d})$ was used for HBV inhibition. Acetylcysteine $(8 \mathrm{~g} / \mathrm{d})$ in $100 \mathrm{ml}$ of $5 \%$ glucose solution and ademetionine $(1 \mathrm{~g} / \mathrm{d})$ in $250 \mathrm{ml}$ of $5 \%$ glucose solution were transfused for liver protection. Besides eating regularly, a $1750 \mathrm{ml}$ solution including $225 \mathrm{~g}$ of glucose, $50 \mathrm{~g}$ of compound amino acids, $10 \mathrm{mg}$ of vitamin B1, $5 \mathrm{mg}$ of vitamin B2, $200 \mathrm{mg}$ of vitamin $\mathrm{C}, 4 \mathrm{~g}$ of potassium chloride and $25 \mathrm{IU}$ of insulin was transfused daily for 20 days against low glucose and hypermetabolism. Dual plasma molecular adsorption system (DPMAS) plus adequate plasma exchange (PE) was performed on the 2nd, 5 th and 8th days of hospitalization, which significantly reduced the serum bilirubin levels. On the 10th day after admission, radioactive iodine $\left({ }^{131} \mathrm{I}\right)$ was applied to treat GD. Levels of FT3 gradually decreased and coagulation function also improved; However, TBil was still at a high level (Fig. 1). Another two DPMAS plus PE therapies were performed on the 12th and 18th days after admission. Ursodeoxycholic acid (UDCA) was taken orally on the 19th day after admission, but ALT, TBil and TBA levels were progressively increased. On the 24th day after admission, laboratory examination showed ALT 96.2 U/L, TBil $440.1 \mu \mathrm{mol} / \mathrm{L}$, TBA $491.8 \mu \mathrm{mol} / \mathrm{L}$, PTA $67 \%$, HBV DNA $20.4 \mathrm{IU} / \mathrm{ml}$ and FT3 $7.1 \mathrm{pmol} / \mathrm{L}$. The patient felt itchy and experienced insomnia. He began to receive prednisone $30 \mathrm{mg}$ orally per day and phenobarbital sodium $50 \mathrm{mg}$ intramuscularly each night. Seven days later, the ALT level was reduced to $68.4 \mathrm{U} / \mathrm{L}$ and TBil was $261.9 \mu \mathrm{mol} / \mathrm{L}$. He stopped taking prednisone for 3 days upon which ALT and TBil levels rebounded instead of decreasing continuously. Prednisone administration $(30 \mathrm{mg} / \mathrm{d})$ was re-instituted and 7 days later, ALT and TBil levels had decreased 
$\mathrm{ETV}+$ Liver protective preparations + Glu and amino acids

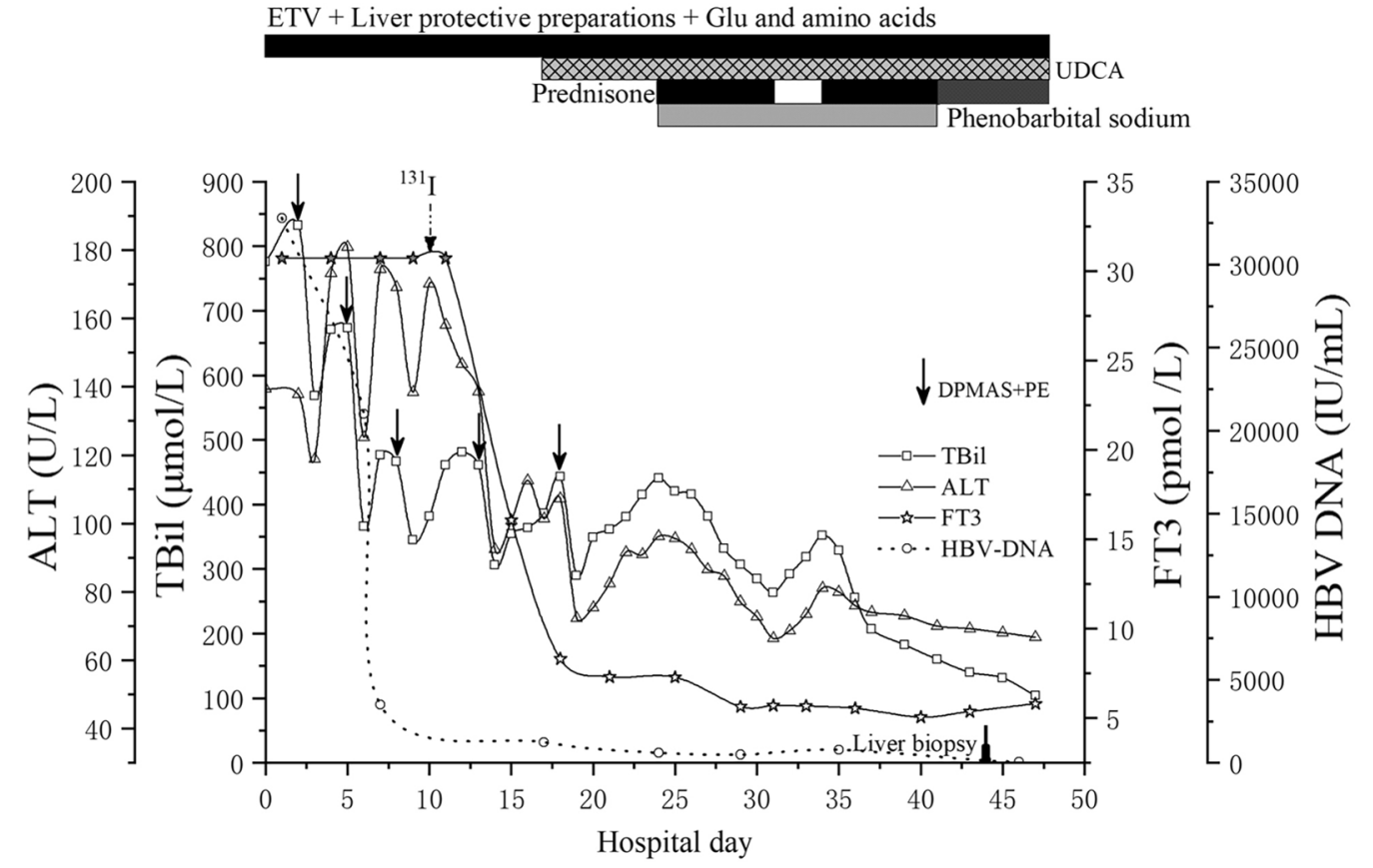

Fig. 1 Laboratory examination results during hospitalization. ALT Alanine transaminase, TBil Total bilirubin, FT3 Free triiodothyronine, HBV DNA Hepatis B virus desoxyribonucleic acid, ETV Entecavir, UDCA Ursodeoxycholic acid, Glu Glucose, DPMAS Dual plasma molecular adsorption system, PE Plasma exchange

and the patient felt no discomfort. Phenobarbital sodium was discontinued and he was treated with a tapering course of oral prednisone. On the 43rd day of hospitalization liver biopsy was performed. Masson trichrome and reticular fiber staining showed partial adjacent portal areas were connected and separate the surrounding hepatic parenchyma and mild interstitial fibrosis; hematoxylin-eosin (HE) staining showed a moderate lymphoplasmacytic infiltration in the portal area, obvious interface hepatitis and hepatocyte rosettes, bile pigment granules in hepatocytes, and bile plugs formation in bile capillaries (Fig. 2). Immunohistochemical staining of CK7 and HBsAg showed moderate proliferative reaction of the periportal bile duct and high expression of HBsAg on the membrane of hepatocytes (data not shown). According to the clinical features and a 7-point RUCAM score and a 6-point simplified IAIHG score (Table 1), the patient was diagnosed as GD overlapping with $\mathrm{CHB}$ and
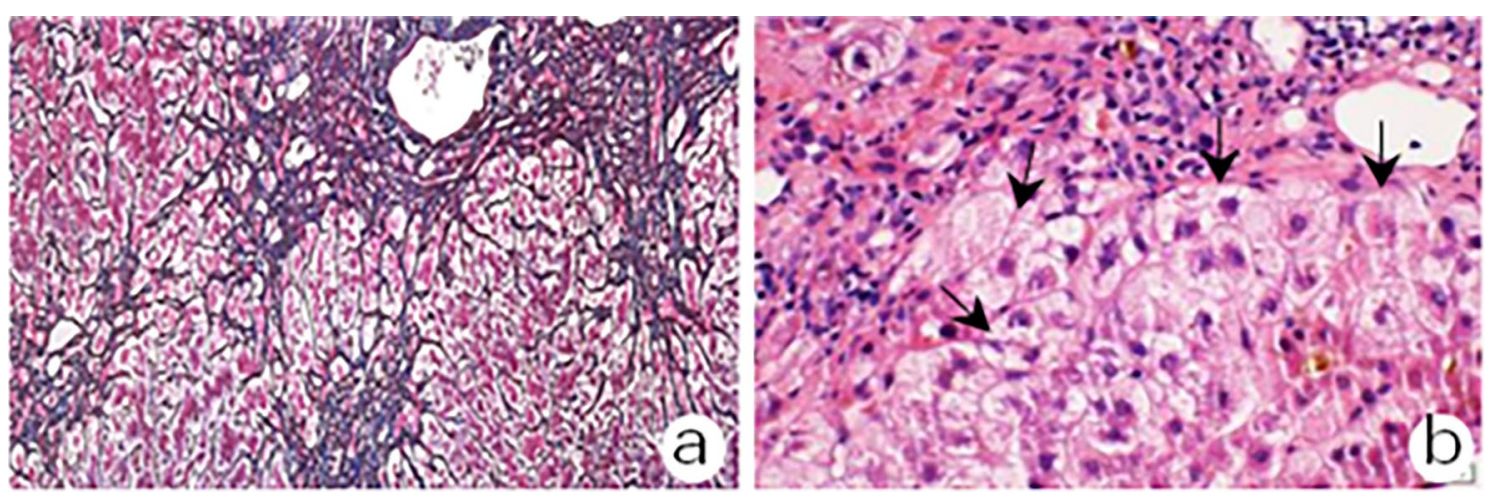

Fig. 2 Laboratory examination results during hospitalization. a Masson trichrome and reticular fiber staining showed partial adjacent portal areas were connected to separate the surrounding hepatic parenchyma and mild interstitial fibrosis; $\mathbf{b}$ HE staining showed a moderate lymphoplasmacytic infiltration in the portal area, obvious interface hepatitis and hepatocyte rosettes (arrow), bile pigment granules in hepatocytes and bile plugs formation in bile capillaries 
Table 1 Autoimmune hepatitis score using simplified scoring system

\begin{tabular}{ll}
\hline Variable & Score \\
\hline ANA $\geq 1: 80$ & 2 \\
IgG>1.1 times ULN* & 2 \\
Liver histology: typical AlH & 2 \\
Absence of viral hepatitis: no & 0 \\
Total & 6 (probable) \\
\hline
\end{tabular}

*Upper limit of normal

MMI-induced liver injury and AIH. On the 47th day of hospitalization, laboratory examination revealed AST $50.3 \mathrm{U} / \mathrm{L}$, ALT $69.1 \mathrm{U} / \mathrm{L}$, TBil 104.7 $\mu \mathrm{mol} / \mathrm{L}$, HBV DNA $10.0 \mathrm{IU} / \mathrm{mL}$, and FT3 $5.81 \mathrm{pmol} / \mathrm{L}$ (Fig. 1). The patient was discharged without discomfort and continued to take ETV $(0.5 \mathrm{mg} / \mathrm{d})$, prednisone $(25 \mathrm{mg} / \mathrm{d} \times 1 \mathrm{w}, 20 \mathrm{mg} / \mathrm{d} \times 2$ $\mathrm{w}, 15 \mathrm{mg} / \mathrm{d} \times 3 \mathrm{w}, 10 \mathrm{mg} / \mathrm{d} \times 4 \mathrm{w})$, ademetionine $(1 \mathrm{~g} / \mathrm{d})$ and UDCA $(500 \mathrm{mg} / \mathrm{d})$. Liver function returned to normal 70 days after discharge, along with a normal level of IgG and ANA. The patient then received a long-term maintenance dose of ETV $0.5 \mathrm{mg} / \mathrm{d}$ after stopping prednisone and UDCA. There was no recurrence during a 6-month follow-up.

\section{Discussion and conclusions}

$\mathrm{CHB}$ is a chronic inflammatory disease of the liver caused by persistent HBV infection, which shows the pathological feature of piecemeal and/or bridging necrosis, infiltration of inflammatory cells and fibrosis. CHB develops insidiously and can only be detected by laboratory examination. Acute exacerbation of chronic hepatitis B (AECHB) involves cytotoxic T cell-mediated cytolysis of HBV antigen(s)-expressing hepatocytes, manifesting as a sudden increase in HBV DNA and an abrupt elevation of ALT to $>5$ ULN or a greater than threefold increase in ALT, whichever is higher [14]. Most cases of AECHB have mild symptoms, but some cases have acute hepatic decompensation or even death [14]. The histopathological characteristic of ACLF caused by AECHB shows massive or submassive liver necrosis [15]. Although the patient had not been tested for HBV previously, he had splenomegaly, an elevation of ALT ( $>5$ ULN), $3.28 \times 10^{4} \mathrm{IU} / \mathrm{mL}$ of HBV DNA, and a decrease of PLT and $\mathrm{CHE}$ after admission. He also had a histopathological feature (G3S3) and an immunocytodiagnostic marker (HBsAg expressing on the membrane of hepatocytes) of CHB. Taken together these findings support a clinical diagnosis of CHB but ACLF, severe intrahepatic cholestasis, periportal bile duct reaction and typical AIH histological features are not the clinical manifestation and pathological characteristics of $\mathrm{CHB}$.
Although GD can induce malnutrition, thyrotoxic hepatitis usually happens when decompensated hyperthyroidism is present [1]. In the last decade, hepatotoxicity and cholestasis induced by MMI have been reported $[3,4,10,15]$. The mechanisms of MMI-associated liver injuries are following: (1) relative hypoxia of the portal system which easily leads to hepatocyte degeneration and necrosis; (2) MMI may aggravate jaundice in the patients with inherited disorders of bilirubin metabolism; (3) several active substances produced in the process of MMI metabolism may damage the hepatocyte and stroma; (4) an allergic reaction [4]. Glucocorticoids are used to effectively treat cholestasis induced by MMI despite the lack of histopathological evidence of the liver $[4,10]$. This patient experienced twice liver injuries when he took MMI for two months one year ago and for 6 days after the second MMI medication. He had a RUCAM score of 7 [16]. Thus, the patient met the diagnostic criteria for MMI-induced liver injury.

The patient also had a simplified IAIHG score of 6 based on a typical AIH histological feature and higher levels of IgG and ANA. Additionally, the elevated ALT and bilirubin rapidly reduced to normal after 3-month glucocorticoid therapy without recurrence during a 6-month follow-up. The patient met the diagnostic criteria for $\mathrm{AIH}$, but which was different from classical $\mathrm{AIH}$ $[17,18]$.

GD together with AIH is a complex and difficult subject to deal with. It contains three forms including GDassociated AIH, GD coexisting with classical AIH and GD complicated with autoimmune-like drug-induced liver injury (AI-DILI), each of which has its own clinical features (Table 2). Briefly, GD-associated AIH was extremely rare and this kind of AIH could be eliminated following a cure of hyperthyroidism [19]. The coexistence of GD with classical AIH is extremely difficult to treat. The combination treatment of long-term steroid use plus anti-thyroid drug or radioactive iodine ablation was indispensable. However, AIH still exists in the case of effective control of hyperthyroidism and the recurrence of AIH may occur if steroid therapy is to be discontinued $[11,20]$. Some of patients with GD coexisting with classical AIH eventually require liver transplantation [11]. Although the pathogenesis of AI-DILI has not yet been fully elucidated, it is generally accepted that the covalent binding of a reactive drug metabolite to a hepatocyte surface protein, formation of a neoantigen, activation of $\mathrm{CD}^{+} \mathrm{T}$ cells with nonselective antigen receptors, and deficient immune regulatory mechanisms are the main bases for a transient loss of self-tolerance, thereby inducing short-term hepatocyte damage [18]. PTU, like other drugs (minocycline, fenofibrate, methyldopa, and statins, and so on), has been reported to induce AI-DILI [21, 22]. 
Table 2 The differences of GD together with AlH

\begin{tabular}{|c|c|c|c|c|c|c|}
\hline Year & Authors & Clinical features & Diagnosis & Therapy & Effect & Ref. no \\
\hline 1999 & Inoue K, et al & $\begin{array}{l}\text { A } 48 \text {-year F suffered from GD and } \\
\text { AlH concurrently. A11 and DR4 are } \\
\text { positive in HLA }\end{array}$ & GD-associated AlH & $\mathrm{MMI}$ & $\begin{array}{l}\text { fT4 and ALT were decreased simul- } \\
\text { taneously after MMI use }\end{array}$ & [19] \\
\hline 2011 & Sato et al & $\begin{array}{l}\text { A 58-year F had three-year GD, and } \\
\text { then PTU-induced Al-DILI }\end{array}$ & GD and PTU- induced AI-DILI & PSL & $\begin{array}{l}\text { Elevated AST level decreased } \\
\text { immediately by PSL }\end{array}$ & {$[22]$} \\
\hline 2019 & Sano et al & $\begin{array}{l}\text { A 15-year F suffered from GD and } \\
\text { treated with MMI. She was ill with } \\
\text { MMI-induced DILI with AlH futures }\end{array}$ & $\begin{array}{l}\text { GD and MMI- induced-DILI } \\
\text { with AlH futures }\end{array}$ & RIA, PSL & $\begin{array}{l}\text { Elevated aminotransferases } \\
\text { couldn't be controlled after RIA but } \\
\text { normalized after one-month PSL }\end{array}$ & {$[11]$} \\
\hline 2019 & Sano et al & $\begin{array}{l}\text { A 21-year F suffered from GD com- } \\
\text { plicated with classical type II AlH }\end{array}$ & GD and type II AlH & RIA, PSL, LT & $\begin{array}{l}\text { AlH couldn't be controlled by RIA } \\
\text { and PSL, and received LT }\end{array}$ & {$[11]$} \\
\hline 2019 & Sano et al & $\begin{array}{l}\text { A 39-year-F suffered from GD com- } \\
\text { plicated with classical AlH }\end{array}$ & GD and $\mathrm{AlH}$ & PSL, RIA, LT & $\begin{array}{l}\text { AlH couldn't be controlled by PSL } \\
\text { and RIA, and received LT }\end{array}$ & {$[11]$} \\
\hline 2019 & Sawhney et al & $\begin{array}{l}\text { A 24-year F was ill with classical } \\
\text { type I AlH, and then GD was found }\end{array}$ & Type I AlH and GD & PSL, AZA, RIA & $\begin{array}{l}\text { AlH be effectively controlled by } \\
\text { the combination of PSL and AZA } \\
\text { and RIA }\end{array}$ & {$[20]$} \\
\hline
\end{tabular}

F female, GD graves' disease, AIH autoimmune hepatitis, AI-DILI autoimmune-like drug-induced liver injury, $M M I$ methimazole, $P T U$ propylthiouracil, $P S L$ prednisone, $R I A$ radioactive iodine ablation, $L T$ liver transplant, $A Z A$ azathioprine

This drug has also been warned by the Food and Drug Administration (FDA) for treating GD in 2016 because of the risk of severe liver injury [2]. The liver injury of GD complicated with PTU-induced AI-DILI was recovered rapidly after one-month steroid therapy [22]. In 2019, Sano S reported a case of GD complicated with MMIinduced liver injury with AIH histological features. The elevated aminotransferase levels of the patient couldn't be controlled after a cure of hyperthyroidism but quickly returned to normal by one-month steroid therapy, implying MMI might be a trigger for AIH [11]. In this study, we reported a case of GD complicated with $\mathrm{CHB}$ and MMIinduced liver injury. Liver biopsy was performed on the 43rd day of hospitalization based on the withdrawal of MMI for eight weeks and effective inhibition of HBV replication for one month and thyroxine returning to normal for two weeks to minimize the influence of other factors (MMI toxicity, HBV replication and hyperthyroidism toxicity) on liver injury. AIH was then identified based on features of typical histology and immunology and good response to steroid, speculating MMI as a possible trigger for AIH. However, we can't exclude the possibility of $\mathrm{HBV}$ as a trigger for AIH $[12,13]$.

The patient met the diagnosis for GD complicated with $\mathrm{CHB}$ and MMI-associated AI-DILI as well as an early stage of ACLF according to his clinical features after admission. Thus, a comprehensive treatment including HBV inhibition, sufficient nutrition, and hepatoprotective drugs and plasma was carried out immediately, which was reasonable. According to the guidelines for diagnosis and management of hyperthyroidism, the use of total/subtotal thyroidectomy was favored in such clinical situations as active
Graves' orbitopathy, patient with periodic paralysis, thyroid malignancy confirmed or suspected, and one of more large thyroid nodules [2]. Although the surgery was also accepted in the situations of coexisting with pregnancy or liver disease or major adverse reactions to anti-thyroid drugs, it was contraindicated in this patient due to liver failure. Therefore, radioactive ${ }^{131} \mathrm{I}$ therapy became the best choice for hyperthyroidism in this case [2]. Since ${ }^{131}$ I therapy can damage thyroid acinar cells to increase the release of the thyroxine in about two weeks and aggravate liver failure, it was difficult to perform this procedure in ACLF. The patient underwent five DPMAS plus PE therapy within 18 days after admission, which sufficiently improved liver failure to be able to safely administer ${ }^{131}$ I treatment on the 10th day of hospitalization. However, serum bilirubin and ALT were still at high levels after effective control of HBV replication and hyperthyroidism. Glucocorticoids have become the first-line treatment for refractory AI-DILI or idiopathic AIH, with a short course of treatment and no recurrence for AI-DILI $[1,23]$. It has been reported to be effective in PTU-induced AI-DILI [1]. In this case, AIH was completely cured by 3 -month steroid therapy without recurrence. However, the exact cause of AIH needs to be further studied.

In summary, we report a case of GD overlapping with $\mathrm{CHB}$ and MMI-induced liver injury and $\mathrm{AIH}$ with an early stage of ACLF. We propose that the combination of $\mathrm{CHB}$ and MMI-induced liver injury and hepatotoxicity of excess thyroxine resulted in ACLF. AIH should be considered when ALT and bilirubin are still at high levels after stopping the culprit drug and gaining effective control of HBV replication and hyperthyroidism. Liver 
biopsy is helpful in the diagnosis of AIH. Glucocorticoids play an important role in the treatment of AIH.

\begin{abstract}
Abbreviations
MMI: Methimazole; GD: Graves' disease; ATP: Adenosine triphosphate; ROS: Reactive oxygen species; ANCA: Antineutrophil cytoplasmic antibody; IAS: Insulin autoimmune syndrome; CHB: Chronic hepatitis B; Al-DILI: Autoimmune-like liver injury; AST: Aspartate aminotransferase; ALT: Alanine aminotransferase; ALP: Alkaline phosphatase; TBil: Total bilirubin; CHE: Cholinesterase; Alb: Albumin; DBil: Direct bilirubin; TBA: Total bile acid; PTA: Prothrombin activity; INR: International normalized ratio; TSH: Thyroid stimulating hormone; FT3: Free triiodothyronine; ULN: Upper limit of normal; TgAb: Anti-thyroglobulin antibody; ANA: Antinuclear antibody; ACLF: Acute-on-chronic liver failure; ETV: Entecavir; DPMAS: Dual plasma molecular adsorption system; PE: Plasma exchange; UDCA: Ursodeoxycholic acid; HE: Hematoxylin-eosin; AECHB: Acute exacerbation of chronic hepatitis B.
\end{abstract}

\section{Acknowledgements}

Declared none.

\section{Authors' contributions}

MFZ conceived the design, performed literature review, and wrote this paper. SYC and WZ performed literature review, DRB revised the manuscript, RPG contributed to the conceptual design, conducted a critical review for intellectual content, read and approved the final version of this paper. All authors read and approved the final manuscript.

\section{Funding}

This work was supported by the Natural Science Foundation of China (81770629) from RPG. RPG contributed to the conceptual design, conducted a critical review for intellectual content, read and approved the final version of this paper.

\section{Availability of data and materials}

The datasets used during the current study are available from the corresponding author on reasonable request.

\section{Declarations}

\section{Ethics approval and consent to participate}

This case study was approved by the Ethics Committee of First Hospital of Jilin University (No. 2021-723).

\section{Consent for publication}

Written informed consent was obtained from the patient for publication of this case report. A copy of the consent form is available for review by the Editor of this journal.

\section{Competing interests}

The authors declare that they have no competing interests.

\section{Author details}

${ }^{1}$ Department of Hepatic Biliary Pancreatic Medicine, First Hospital of Jilin University, 71 Xinmin Avenue, Changchun 130021, Jilin, China. ${ }^{2}$ The Research Institute at Nationwide Children's Hospital, Columbus, OH, USA.

Received: 18 April 2021 Accepted: 1 February 2022

Published online: 10 February 2022

\section{References}

1. de Campos Mazo DF, de Vasconcelos GB, Pereira MA, de Mello ES, Bacchella T, Carrilho FJ, et al. Clinical spectrum and therapeutic approach to hepatocellular injury in patients with hyperthyroidism. Clin Exp Gastroenterol. 2013;6:9-17.

2. Ross DS, Burch HB, Cooper DS, Greenlee MC, Laurberg P, Maia AL, et al. 2016 American thyroid association guidelines for diagnosis and management of hyperthyroidism and other causes of thyrotoxicosis. Thyroid. 2016;26(10):1343-421.

3. López PRP, Forero JD, Sierra F. Methimazole-induced cholestatic jaundice in a hyperthyroid patient. Acta Gastroenterol Latinoam. 2014;44(1):52-8.

4. Zou H, Jin L, Wang LR, Braddock M, Cai WW, Zheng MH. Methimazoleinduced cholestatic hepatitis: two cases report and literature review. Oncotarget. 2016;7(4):5088-91.

5. Yang J, Li LF, Xu Q, Zhang J, Weng WW, Zhu YJ, et al. Analysis of 90 cases of antithyroid drug-induced severe hepatotoxicity over 13 years in China. Thyroid. 2015;25(3):278-83.

6. Niknahad H, Jamshidzadeh A, Heidari R, Hosseini Z, Mobini K, Khodaei $F$, et al. Paradoxical effect of methimazole on liver mitochondria: in vitro and in vivo. Toxicol Lett. 2016;259:108-15.

7. Li X, Yang J, Jin S, Dai Y, Fan Y, Fan X, et al. Mechanistic examination of methimazole-induced hepatotoxicity in patients with Grave's disease: a metabolomic approach. Arch Toxicol. 2020:94(1):231-44.

8. Bonaci-Nikolic B, Nikolic MM, Andrejevic S, Zoric S, Bukilica M. Antineutrophil cytoplasmic antibody (ANCA)-associated autoimmune diseases induced by antithyroid drugs: comparison with idiopathic ANCA vasculitides. Arthritis Res Ther. 2005:7(5):R1072-81.

9. Han R, Jiang X. Methimazole-induced insulin autoimmune syndrome in Graves' disease with hypokalemia: a case report and literature review. Exp Ther Med. 2020;20(5):93.

10. Zhang M, Zhou H, He R, Di F, Yang L, Yang T. Steroids for the treatment of methimazole-induced severe cholestatic jaundice in a 74-year-old woman with type 2 diabetes. Endocrine. 2010;37(2):241-3.

11. Rana S, Ahmed Z, Salgia R, Bhan A. Successful management of patients with co-existent graves' disease and autoimmune hepatitis. Cureus. 2019:11(5):e4647.

12. Maya R, Gershwin ME, Shoenfeld Y. Hepatitis B virus (HBV) and autoimmune disease. Clin Rev Allergy Immunol. 2008;34(1):85-102.

13. Sostre V, Patel HG, Mohamed A, Volfson A. A case of acute autoimmune hepatitis superimposed on chronic hepatitis B infection. Case Rep Gastrointest Med. 2018;2018:2139607.

14. Tsai WL, Sun WC, Cheng JS. Chronic hepatitis B with spontaneous severe acute exacerbation. Int J Mol Sci. 2015;16(12):28126-45.

15. Sarin SK, Choudhury A, Sharma MK, Maiwall R, Al Mahtab M, Rahman S, et al. Acute-on-chronic liver failure: consensus recommendations of the Asian Pacific association for the study of the liver (APASL): an update. Hepatol Int. 2019:13(4):353-90.

16. Teschke R, Zhu Y, Jing J. Herb-induced liver injury in Asia and current role of RUCAM for causality assessment in 11,160 published cases. J Clin Trans Hepatol. 2020;8(2):200-14.

17. Hennes EM, Zeniya M, Czaja AJ, Parés A, Dalekos GN, Krawitt EL, et al. Simplified criteria for the diagnosis of autoimmune hepatitis. Hepatology. 2008;48(1):169-76.

18. Czaja AJ. Drug-induced autoimmune-like hepatitis. Dig Dis Sci. 2011;56(4):958-76.

19. Inoue K, Okajima T, Tanaka E, Ando B, Takeshita M, Masuda A, et al. A case of Graves' disease associated with autoimmune hepatitis and mixed connective tissue disease. Endocr J. 1999:46(1):173-7.

20. Sawhney R, Dhingra S, Sood GK. Autoimmune hepatitis refractory to treatment due to underlying Grave's disease. Cureus. 2019;11(5):e4598.

21. Stine JG, Chalasani N. Chronic liver injury induced by drugs: a systematic review. Liver Int. 2015:35(11):2343-53.

22. Sato I, Tsunekawa T, Shinohara Y, Nishio Y, Shimizu Y, Suzuki Y, et al. A case of autoimmune hepatitis with Graves' disease treated by propylthiouracil. Nagoya J Med Sci. 2011;73(3-4):205-9.

23. Björnsson ES, Bergmann O, Jonasson JG, Grondal G, Gudbjornsson B, Olafsson S. Drug-induced autoimmune hepatitis: response to corticosteroids and lack of relapse after cessation of steroids. Clin Gastroenterol Hepatol. 2017;15(10):1635-6.

\section{Publisher's Note}

Springer Nature remains neutral with regard to jurisdictional claims in published maps and institutional affiliations. 